\title{
Clinical and molecular characteristics associated with the efficacy of PD-I/PD-LI inhibitors for solid tumors: a meta-analysis
}

This article was published in the following Dove Press journal:

OncoTargets and Therapy

\section{Yi Ming Weng \\ Min Peng \\ Meng Xue Hu \\ Yi Yao \\ Qi Bin Song}

Department of Oncology, Renmin Hospital of Wuhan University, Wuhan University, Wuhan, Hubei, People's Republic of China
Correspondence: Qi Bin Song Department of Oncology, Renmin Hospital of Wuhan University, Jiefang Road, Wuchang Qu, Wuhan Shi, Hubei Sheng 430072, People's Republic of China Email qibinsong@163.com

\begin{abstract}
We conducted a meta-analysis to estimate the impact of different clinical and molecular characteristics on the efficacy of programmed cell death 1 (PD-1) or programmed cell death ligand 1 (PD-L1) inhibitors. PubMed and Web of Science were searched for related trials. Eleven eligible studies, comprising 5,663 patients, were included in this meta-analysis. We found that the PD-1/PD-L1 inhibitor was associated with a $31 \%$ reduction in the risk of death (hazard ratio $[\mathrm{HR}]=0.69 ; 95 \%$ CI $0.64-0.74 ; P<0.00001$ ) for patients with melanoma, non-small-cell lung cancer (NSCLC), urothelial carcinoma, head and neck carcinoma, and renal cell carcinoma. In subgroup analyses, all the patients with PD-L1-positive tumors had overall survival (OS) benefits from PD-1/PD-L1 inhibitors regardless of PD-L1 expression level, and a dose-effect relationship between the expression of PD-L1 and OS benefit from PD-1/PD-L1 inhibitors was observed. There was an OS improvement for patients with a smoking history $(P<0.00001)$, but no OS benefit was observed for nonsmokers $(P=0.28)$. In addition, first-line therapy had better OS than second-line or later treatment $(P=0.02)$. No significant improvement of OS was observed $(P=0.70)$ in patients aged $\geq 75$ years. The relative treatment efficacy was similar according to sex (male vs female, $P=0.60$ ), performance status ( 0 vs $\geq 1, P=0.68$ ), tumor histology (squamous NSCLC vs non-squamous NSCLC vs melanoma vs urothelial carcinoma vs head and neck carcinoma vs renal cell carcinoma, $P=0.64$ ), and treatment type (PD-1 inhibitor vs PD-L1 inhibitor, $P=0.36$ ). In conclusion, PD-L1-positive tumors, smoking history, and first-line treatment were potential factors for the efficacy of PD-1/PD-L1 inhibitors. Patients with higher PD-L1 expression might achieve greater OS benefits. In addition, sex, performance status, tumor histology, and treatment type could not predict the efficacy of this therapy. In contrast, patients aged $>75$ years and nonsmokers might not get OS benefits from this treatment. These results may improve treatment strategies and patient selection for PD-1/PD-L1 inhibitors.
\end{abstract}

Keywords: anti-programmed cell death 1, biomarker, solid tumor, meta-analysis

\section{Introduction}

Cancer is one of the leading causes of death worldwide. Surgery, chemotherapy, and radiotherapy have been widely used as standard treatments for cancer patients. However, the overall survival (OS) rates of patients are still far from ideal. Cancer can be thought of as a host's inability to eliminate transformed cells. Cancer immunotherapy refers to a diverse range of therapeutic methods that harness the immune system to induce or restore the capacity of cytotoxic T cells, and other immune effector cells, and to recognize and eliminate cancer. ${ }^{1}$ Among many immunotherapeutic strategies, immune checkpoint inhibitor (ICI), which directly restores the efficacy of 
tumor-specific T cells within the tumor microenvironment, thereby enhancing the capacity of immune system to fight cancers, has shown remarkable benefit in the treatment of a range of cancer types. ${ }^{2}$ Programmed cell death receptor- 1 (PD-1) and programmed cell death ligand-1 (PD-L1) are the most widely studied and recognized inhibitory checkpoint pathways. Several clinical trials using inhibitors blocking these pathways for the treatment of malignancies, such as melanoma, non-small-cell lung cancer (NSCLC), head and neck cancer, renal cell cancer, urothelial cancer, and lymphoma, have shown great promise in prolonging survival. ${ }^{3-5}$ The US Food and Drug Administration (FDA) has approved five PD-1/PD-L1 inhibitors in eleven types of advanced malignancies. ${ }^{6}$

Although promising results of PD-1/PD-L1 inhibitors have been observed in major clinical studies, around $40 \%-60 \%$ of patients still do not benefit from these therapies. ${ }^{3}$ In addition, these treatments are associated with immune-related adverse events, such as dermatologic (pruritus, rash), gastrointestinal (diarrhea, colitis), hepatic (elevated liver enzymes), and endocrine (pituitary, thyroid, adrenal glands) complications and life-threatening adverse events. ${ }^{7}$ In the CheckMate-067 trial, severe immune-related adverse events (grades 3 or 4) were observed in 55\% patients treated with nivolumab plus ipilimumab: $16 \%$ in the nivolumab monotherapy group and $27 \%$ in the ipilimumab monotherapy group. ${ }^{8}$ In the new era of precision medicine, identifying biomarkers that can predict the benefit of ICIs is crucial to protect patients from autoimmune adverse effects and the high cost of such agents. Currently, PD-L1 expression has emerged as a biomarker that might help to predict responses to PD-1/PD-L1 inhibitors. Companion tests for evaluating PD-L1 expression as a biomarker of response have been developed for many cancer immunotherapy agents. However, PD-L1 assays can be highly variable, which makes it a clinical challenge to employ the results. ${ }^{9,10}$ In addition, because of the complexity of the immune response and tumor biology, it is unlikely that a single biomarker will be sufficient to predict clinical outcomes in response to immunotargeted therapy. Thus, the integration of multiple clinical and molecular characteristics may be necessary for the accurate prediction of the clinical benefit of PD-1/PD-L1 inhibitors.

A previous meta-analysis determined that there was an OS advantage of PD-1/PD-L1 inhibitors for patients with EGFR wild-type NSCLC, and no OS advantage was observed for those with EGFR-mutant tumors. However, PD-L1 expression and some other factors were not analyzed in the previous study, because of an insufficient number of trials that reported those results. ${ }^{11} \mathrm{With}$ accumulating evidence, in this meta-analysis, we systematically evaluated the current evidence regarding biomarkers of response to PD-1/PD-L1 inhibitors in all solid tumors. The identification of predictive clinical and molecular characteristic factors may be valuable for clinical practice and future clinical study design.

\section{Materials and methods}

We conducted this meta-analysis based on the Preferred Reporting Items for Systematic Reviews and Meta-analyses (PRISMA) statement. All analyses were based on previous published studies; thus, no ethical approval and patient consent are required.

\section{Search strategy}

Two reviewers independently completed a search of PubMed and Web of science to identify relevant clinical trials. The search was conducted using the following keywords: "nivolumab", "pembrolizumab", "atezolizumab", "avelumab", "durvalumab", and "immune checkpoint". The search was limited to randomized controlled trials (RCTs) published in English. No other limitation was used (ie, ethnic background and peer review publication only). References of included studies and related reviews were checked manually. If the results of RCTs were published in a series of articles, only the most recent, complete report of the clinical trial was included for analysis.

\section{Inclusion criteria}

All relevant articles underwent evaluation for eligibility by two investigators independently. The relevant clinical trials were selected carefully based on the following criteria: 1) population: participants with histologically confirmed solid tumors; 2) intervention: PD-1 or PD-L1 inhibitors; 3) comparison: chemotherapy or cytotoxic T-lymphocyte antigen-4 (CTLA-4) inhibitor; 4) outcomes: OS; and 5) study design: RCTs.

\section{Data extraction and quality assessment}

Literature screening and data extraction were carried out by two independent reviewers. Discrepancy was resolved by discussion between the two of us. If the two authors could not reach a consensus, another author made the decision. For each study, the following details were extracted: the name of first author, year of publication, cancer type, treatment arms and control arms, patients' clinicopathological characteristics. We also extracted hazard ratio (HR) and 95\% CI for OS (defined as the time from randomization to death) of the intention-to-treat population and the following predefined subgroups: age ( $<65$ vs $\geq 65$ years), sex (female vs male), 
performance status ( $\mathrm{PS}=0 \mathrm{vs} \geq 1$ ), line of therapy (one line vs $\geq 2$ line), smoking status (never smokers vs ever smokers), tumor histology (squamous NSCLC vs non-squamous NSCLC vs melanoma), treatment type (PD-1 inhibitor vs PD-L1 inhibitor), and PD-L1 expression ( $<1 \%$ vs $\geq 1 \%$ vs $<5 \%$ vs $\geq 5 \%$ vs $<10 \%$ vs $\geq 10 \%$ vs $\geq 50 \%$ ).

\section{Statistical analyses}

Statistical analyses were performed with Review Manager, version 5.1 (The Nordic Cochrane Centre, Copenhagen, Denmark). Statistical heterogeneity between different trials was assessed by Cochran's Q statistic. Heterogeneity was considered statistically significant when $P<0.05$. If heterogeneity was present, the data were analyzed by a random-effects model; otherwise, a fixed-effects model was used. To explore the potential sources of heterogeneity, the sensitivity analysis was performed in this review. Potential publication biases were estimated by funnel plots, Begg's test, and Egger's test. Pooled HR for OS with 95\% CI was calculated by the proper algorithm. $P<0.05$ was regarded as statistically significant, and all $P$-values were two sided.

\section{Results Study characteristics}

Studies were identified as shown in Figure 1. In all studies, this meta-analysis included eleven RCTs with 3,090 patients in the experimental group and 2,573 in the control group. ${ }^{12-22}$ Characteristics of included trials are listed in Table 1. Five studies evaluated nivolumab monotherapy vs chemotherapy, three studies assessed pembrolizumab monotherapy vs chemotherapy, two studies evaluated atezolizumab vs chemotherapy, and one study evaluated nivolumab/ipilimumab combination vs ipilimumab. Two trials enrolled melanoma patients, six enrolled NSCLC patients, one enrolled urothelial carcinoma patients, one enrolled head and neck carcinoma patients, and one study enrolled renal cell carcinoma patients. Eight trials reported the ethnic background of patients, and all these studies consisted primarily of Caucasians and small number of Asians.

\section{Impact of PD-I/PD-LI inhibitors on OS}

All the studies, which collectively included 5,663 patients, reported OS data. Of the eleven trials, ten reported a statistically significant improvement in OS and one trial

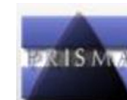

PRISMA 2009 flow diagram
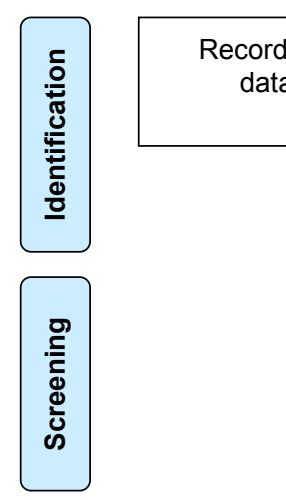

Additional records identified through other sources (references of included studies and related reviews) database searching $(n=657)$ $(n=6)$
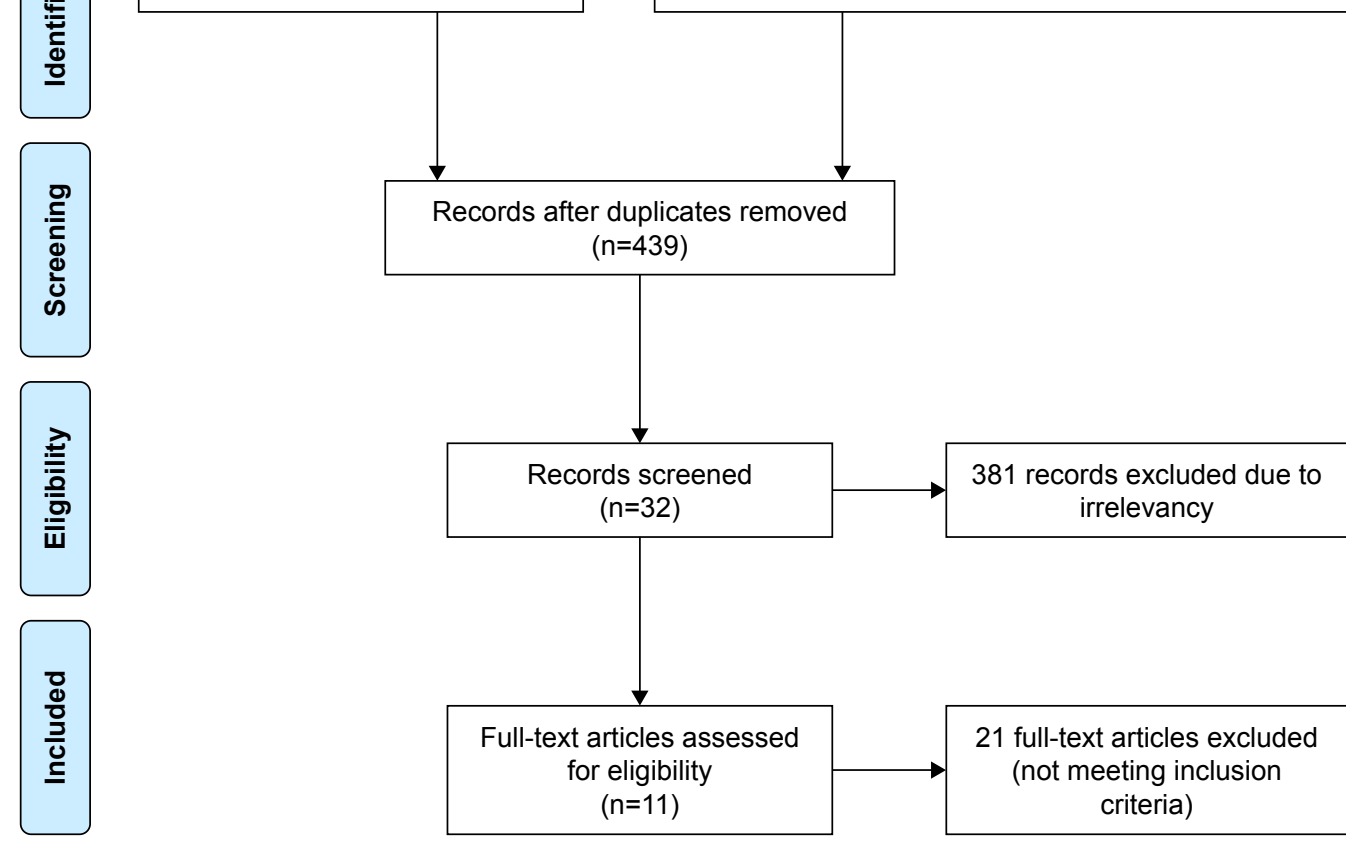

Figure I Flowchart of the literature search and study selection procedure.

Notes: Adapted from Moher D, Liberati A, Tetziaff J, Altman DG, The PRISMA Group (2009). Preferred Reporting Items for Systematic Reviews and Meta-Analyses. The PRISMA Statement. PLoS Med. 6(7):el 000097. doi:I0.137I/journal.pmed I000097.43

Abbreviation: PRISMA, Preferred Reporting Items for Systematic Reviews and Meta-analyses. 
Table I Characteristics of patients in the included trials

\begin{tabular}{|c|c|c|c|c|c|c|c|c|}
\hline Study & Cancer type & $\begin{array}{l}\text { Treatment } \\
\text { comparison }\end{array}$ & Case & $\operatorname{Sex}(n)$ & $\begin{array}{l}\text { Age } \\
\text { (years) }\end{array}$ & $\begin{array}{l}\text { PS } \\
\text { (n) }\end{array}$ & $\begin{array}{l}\text { Smoke } \\
\text { (n) }\end{array}$ & CNS \\
\hline \multirow[t]{4}{*}{ Bellmunt et $\mathrm{al}^{13}$} & Urothelial & Pembrolizumab & 270 vs & Female 140 & $<65$ & 0 or 1 & No & - \\
\hline & carcinoma & vs chemotherapy & 272 & & 230 & 526 & 187 & \\
\hline & & & & Male 402 & $\geq 65$ & 2 & Yes & - \\
\hline & & & & & 312 & 6 & 67 & \\
\hline \multirow[t]{7}{*}{ Borghaei et al ${ }^{2 !}$} & Non-squamous & Nivolumab vs & 292 vs & Female 263 & $<65$ & 0 & No & Yes \\
\hline & NSCLC & docetaxel & 290 & & 339 & 179 & 118 & 68 \\
\hline & & & & Male 319 & $\geq 65$ & I & Yes & No \\
\hline & & & & & $<75$ & 401 & 458 & 514 \\
\hline & & & & & 200 & & & \\
\hline & & & & & $\geq 75$ & & & \\
\hline & & & & & 43 & & & \\
\hline \multirow[t]{7}{*}{ Brahmer et al ${ }^{19}$} & Squamous & Nivolumab vs & 135 vs & Female 64 & $<65$ & 0 & No & Yes \\
\hline & NSCLC & docetaxel & 137 & & 152 & 64 & 17 & 17 \\
\hline & & & & Male 208 & $\geq 65$ & 1 & Yes & No \\
\hline & & & & & $<75$ & 206 & 250 & 255 \\
\hline & & & & & 91 & & & \\
\hline & & & & & $\geq 75$ & & & \\
\hline & & & & & 29 & & & \\
\hline \multirow[t]{4}{*}{ Fehrenbacher et $\mathrm{al}^{18}$} & NSCLC & Atezolizumab vs & I44 vs & Female II8 & - & 0 & No & - \\
\hline & & docetaxel & 143 & & & 91 & 56 & \\
\hline & & & & Male 169 & - & 1 & Yes & - \\
\hline & & & & & & 193 & 231 & \\
\hline \multirow[t]{4}{*}{ Ferris et a $\left.\right|^{14}$} & Head and neck & Nivolumab vs & 240 vs & Female 6I & - & 0 & No & - \\
\hline & carcinoma & chemotherapy & 121 & & & 72 & 70 & \\
\hline & & & & Male 300 & - & 1 & Yes & - \\
\hline & & & & & & 283 & 276 & \\
\hline \multirow[t]{4}{*}{ Herbst et al ${ }^{17}$} & NSCLC & Pembrolizumab & 690 vs & Female 399 & $<65$ & 0 & No & Yes \\
\hline & & vs docetaxel & 343 & & 604 & 348 & 190 & 152 \\
\hline & & & & Male 634 & $\geq 65$ & I & Yes & No \\
\hline & & & & & 429 & 678 & 833 & 881 \\
\hline \multirow[t]{4}{*}{ Hodi et al ${ }^{15}$} & Melanoma & Nivolumab + & 95 vs & Female 47 & $<65$ & 0 & - & Yes \\
\hline & & ipilimumab & 47 & & 68 & 116 & & 4 \\
\hline & & Ipilimumab & & Male 95 & $\geq 65$ & I & - & No \\
\hline & & & & & 74 & 24 & & 137 \\
\hline \multirow[t]{7}{*}{ Motzer et al ${ }^{20}$} & Renal cell & Nivolumab vs & 410 vs & Female 202 & $<65$ & - & - & - \\
\hline & carcinoma & everolimus & $4 I I$ & & 497 & & & \\
\hline & & & & Male 619 & $\geq 65$ & - & - & - \\
\hline & & & & & $<75$ & & & \\
\hline & & & & & 250 & & & \\
\hline & & & & & $\geq 75$ & - & - & - \\
\hline & & & & & 74 & & & \\
\hline \multirow[t]{4}{*}{ Reck et al ${ }^{16}$} & NSCLC & Pembrolizumab & I54 vs & Female 118 & - & 0 & No & Yes \\
\hline & & vs chemotherapy & $|5|$ & & & 107 & 24 & 28 \\
\hline & & & & Male 187 & - & I & Yes & No \\
\hline & & & & & & 197 & 281 & 277 \\
\hline \multirow[t]{4}{*}{ Rittmeyer et al ${ }^{12}$} & NSCLC & Atezolizumab vs & 450 vs & Female 330 & $<65$ & 0 & No & Yes \\
\hline & & docetaxel & 450 & & 453 & 315 & 156 & 85 \\
\hline & & & & Male 520 & $>65$ & I & Yes & No \\
\hline & & & & & 397 & 535 & 694 & 765 \\
\hline \multirow[t]{7}{*}{ Robert et al $\left.\right|^{22}$} & Melanoma & Nivolumab vs & $210 \mathrm{vs}$ & Female 172 & $<65$ & 0 & - & Yes \\
\hline & & dacarbazine & 208 & & 200 & 269 & & 15 \\
\hline & & & & Male 246 & $\geq 65$ & I & - & No \\
\hline & & & & & $<75$ & 144 & & 403 \\
\hline & & & & & $|5|$ & & & \\
\hline & & & & & $\geq 75$ & & & \\
\hline & & & & & 67 & & & \\
\hline
\end{tabular}




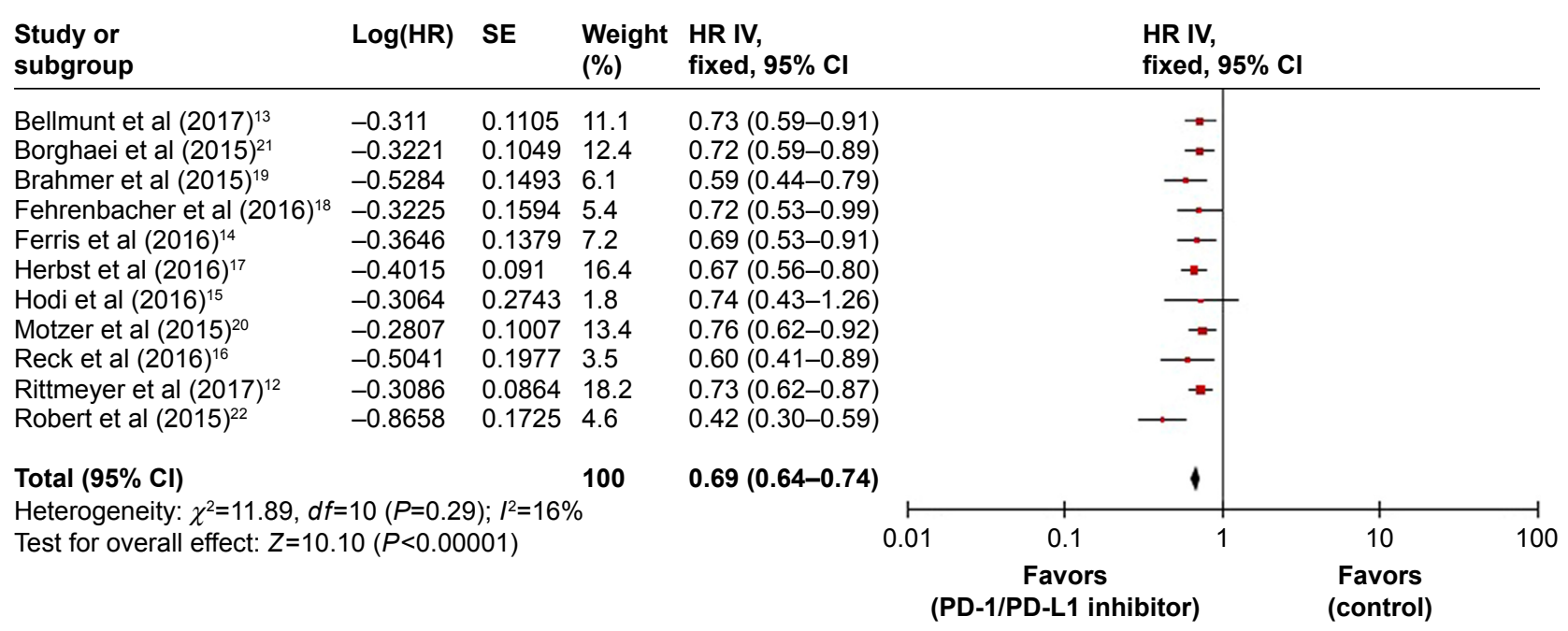

Figure 2 Forest plot for the meta-analysis of HR of OS for PD-I or PD-LI inhibitors in solid tumors.

Abbreviations: HR, hazard ratio; OS, overall survival; PD-I, programmed cell death I; PD-LI, programmed cell death ligand-I.

showed no OS benefit. The median OS in the PD-1/PD-L1 inhibitor groups reported in eleven trials ranged from 7.5 to 25.0 months. The heterogeneity across the eleven trials was insignificant $\left(P=0.29, I^{2}=16 \%\right.$ ). The pooled HR for OS was performed using a fixed-effects model. In pooled analyses, compared with chemotherapy or CTLA-4 inhibitor, PD-1/ PD-L1 inhibitor was significantly associated with a $31 \%$ reduction in the risk of death $(\mathrm{HR}=0.69 ; 95 \%$ CI $0.64-0.74$; $P<0.00001$; Figure 2).

\section{Subgroup analyses by PD-LI expression}

All the studies analyzed the correlation between the PD-L1 expression level ( $<1 \%$ vs $\geq 1 \%,<5 \%$ vs $\geq 5 \%,<10 \%$ vs $\geq 10 \%$ and $\geq 50 \%$ ) and OS benefit of PD-1/PD-L1 inhibitors: PD-L1 $<5 \%$ population including the PD-L1 $<1 \%$ population and $\mathrm{PD}-\mathrm{L} 1 \geq 1 \%$ population including the PD-L1 $\geq 5 \%$ population.

For the PD-L $1<1 \%$ population, seven studies reported OS data. Four trials enrolled NSCLC patients, one enrolled urothelial carcinoma patients, one enrolled head and neck carcinoma patients, and one enrolled renal cell carcinoma patients. Statistically significant improvements in OS were observed in three trials. The heterogeneity between studies was insignificant $\left(P=0.62, I^{2}=0 \%\right.$ ). A fixed-effects model was used to pool HR for OS. The pooled HR for OS was 0.81 (95\% CI $0.71-0.91 ; P=0.0005$ ). The PD-L1 $<1 \%$ population could get OS benefits from PD-1/PD-L1 inhibitor therapy. For the PD-L1 $\geq 1 \%$ population, OS data were available for seven studies, which are same as the PD-L1 $<1 \%$ population. There was no significant heterogeneity $\left(P=0.76, I^{2}=0 \%\right)$, and a fixed-effects model was used to pool HR for OS. The PD-1/PD-L1 inhibitor therapy significantly improved OS for the $\mathrm{PD}-\mathrm{L} 1 \geq 1 \%$ population $(\mathrm{HR}=0.66,95 \%$ CI $0.58-0.75$; $P<0.00001)$. The result of test for subgroup differences showed that the PD-L1 $\geq 1 \%$ population had better OS than the PD-L $1<1 \%$ population $\left(P=0.02, I^{2}=80.7 \%\right.$; Figure $\left.3 \mathrm{~A}\right)$.

For the $\mathrm{PD}-\mathrm{L} 1<5 \%$ population, five studies were included in the analysis, and substantial heterogeneity was observed $\left(P=0.04, I^{2}=59 \%\right)$. The pooled HR using a random-effects model for OS was 0.74 (95\% CI 0.56-0.98; $P=0.04)$. The PD-L1 $<5 \%$ population could get OS benefits from PD-1/ PD-L1 inhibitor therapy. For the PD-L1 $\geq 5 \%$ population, seven clinical trials reported OS data. No significant heterogeneity was found $\left(P=0.38, I^{2}=6 \%\right)$. PD-1/PD-L1 inhibitor therapy significantly improved OS for the PD-L1 $\geq 5 \%$ population $(\mathrm{HR}=0.53,95 \% \mathrm{CI} 0.44-0.63 ; P<0.00001)$. Compared with the $\mathrm{PD}-\mathrm{L} 1<5 \%$ population, there was a tendency for PD-1/PD-L1 inhibitor to improve OS in the PD-L1 $\geq 5 \%$ population $\left(P=0.05, I^{2}=74.8 \%\right.$; Figure $\left.3 \mathrm{~B}\right)$.

For the $\mathrm{PD}-\mathrm{L} 1<10 \%$ population, four studies reported OS data. Three trials enrolled NSCLC patients and one enrolled urothelial carcinoma patients. No significant heterogeneity was observed $\left(P=0.02, I^{2}=5 \%\right)$. The pooled $\mathrm{HR}$ for OS was performed using a fixed-effects model. PD-1/PD-L1 inhibitor therapy significantly improved OS for the PD-L $1<10 \%$ population $(\mathrm{HR}=0.82,95 \% \mathrm{CI} 0.70-0.97 ; P=0.02)$. For the PD-L1 $\geq 10 \%$ population, four trials were included in the analysis, and heterogeneity was not obvious. The pooled HR for OS was 0.49 (95\% CI $0.38-0.62 ; P<0.00001)$. The PD-L1 $\geq 10 \%$ population could get OS benefits from PD-1/ PD-L1 inhibitor therapy. The result of test for subgroup differences showed that the PD-L1 $\geq 10 \%$ population had better OS than the PD-L $1<10 \%$ population $\left(P=0.0004, I^{2}=92.1 \%\right.$; Figure 3C). 
For the PD-L1 $\geq 50 \%$ population, four studies were included in the analysis. Four trials enrolled NSCLC patients. There was no significant heterogeneity between the four studies $(P=0.65$, $I^{2}=0 \%$ ). The $\mathrm{PD}-\mathrm{L} 1 \geq 50 \%$ population got significant improvement in OS from PD-1/PD-L1 inhibitor therapy (HR $=0.52$, 95\% CI 0.43-0.63; $P<0.00001$; Figure 3D).

\section{Subgroup analyses by patient factors}

Subgroup analyses were conducted based on patient factors such as smoking status, age, PS, and sex. In the ever-smoked (current or former) subgroup, five studies reported OS data. Four trials enrolled NSCLC patients and one enrolled urothelial carcinoma patients. The pooled HR for OS was

A

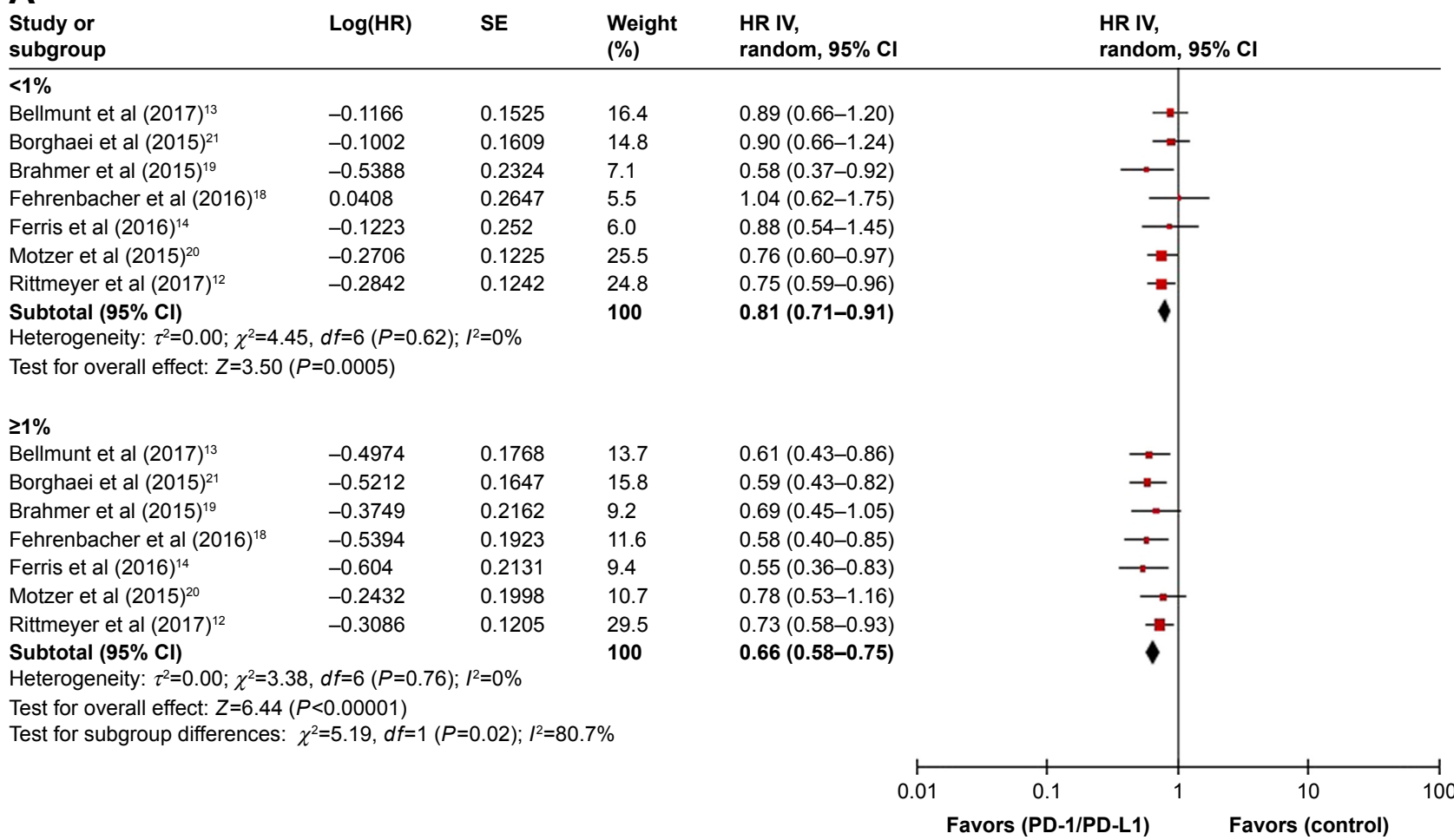

B

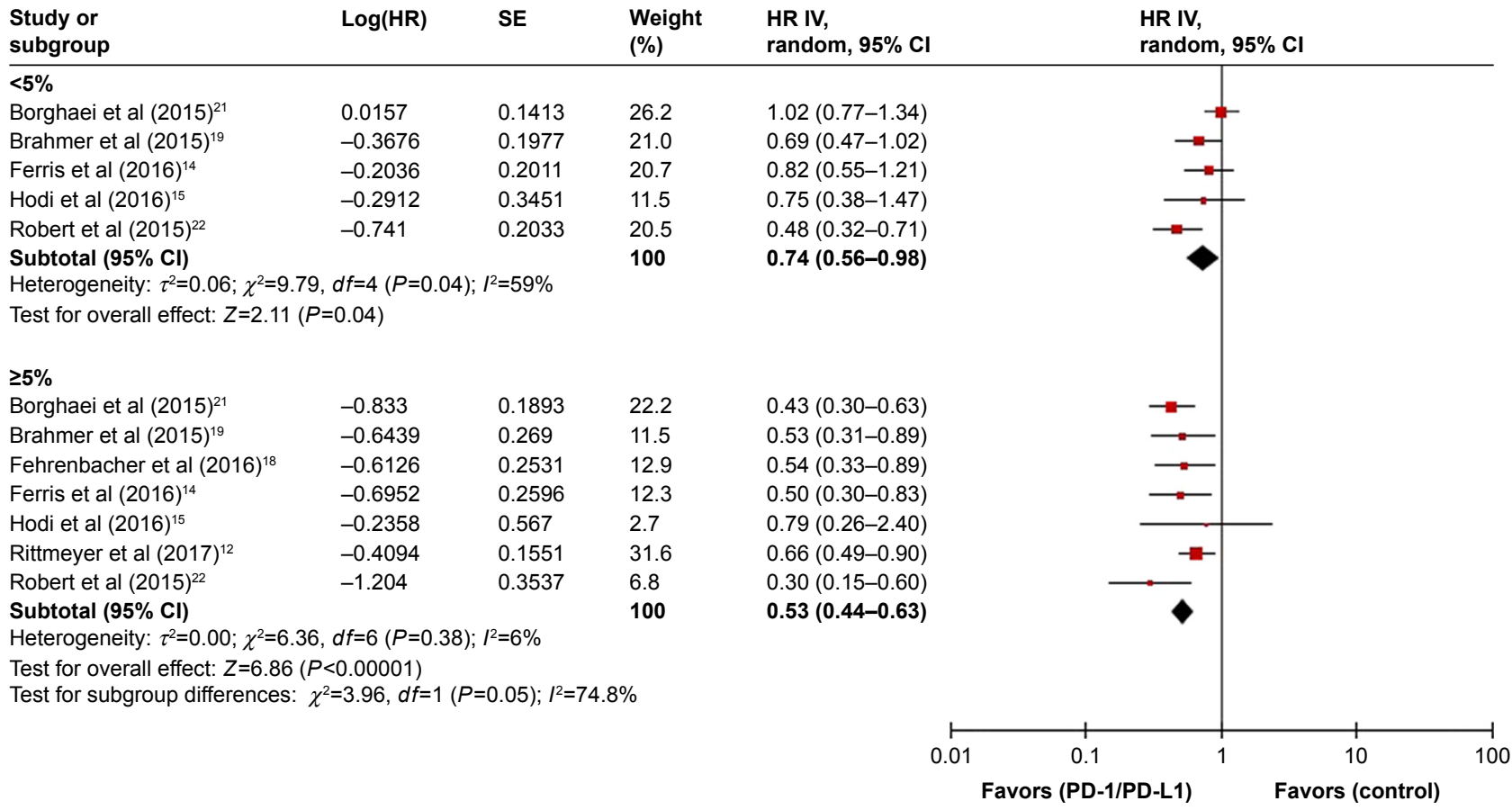

Figure 3 (Continued) 


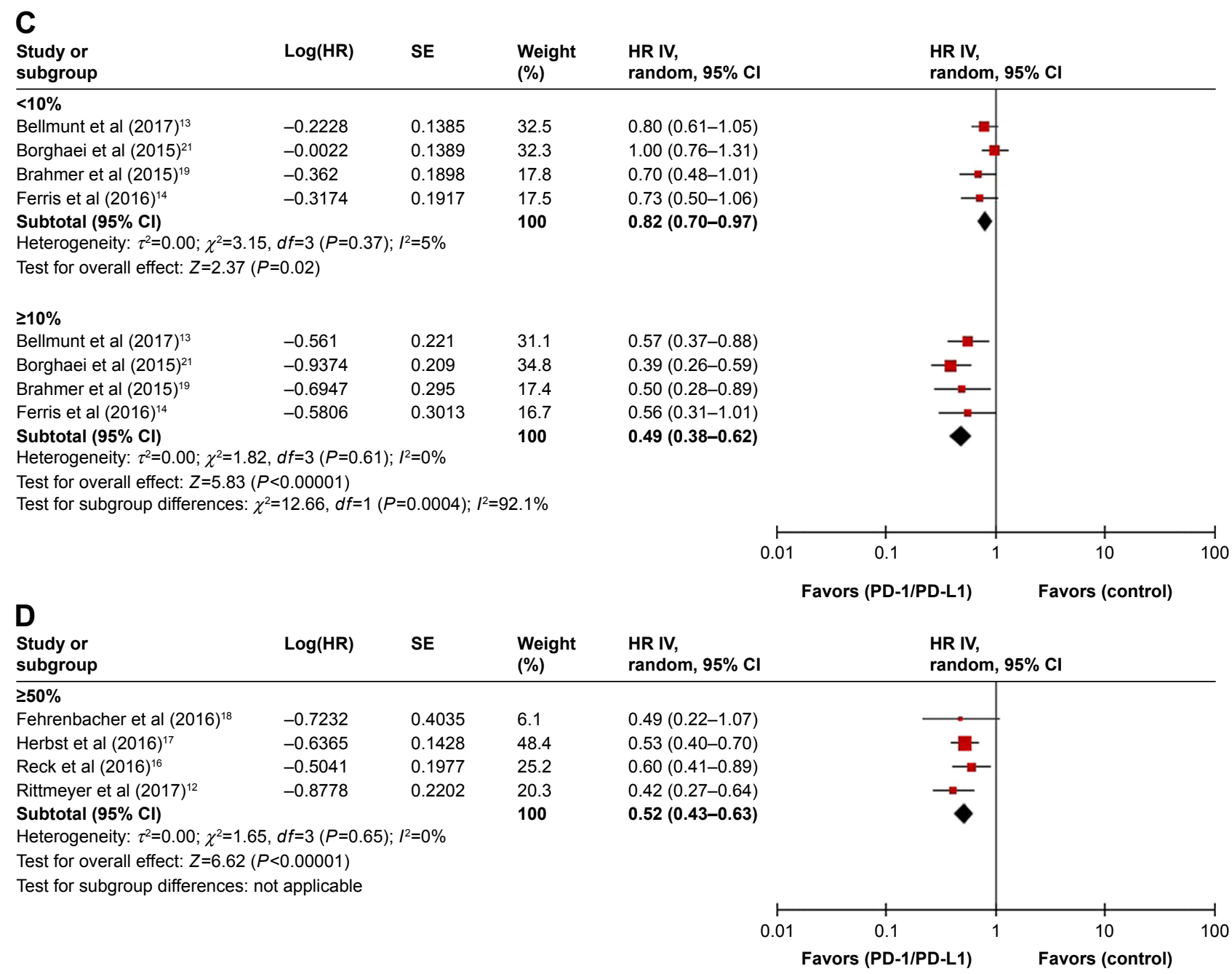

Figure 3 Forest plot for the subgroup analyses of HR of OS for PD-I or PD-LI inhibitors by PD-LI expression.

Notes: (A) PD-LI expression $<1 \%$ vs PD-LI expression $\geq 1 \%$. (B) PD-LI expression $<5 \%$ vs PD-LI expression $\geq 5 \%$. (C) PD-LI expression $<10 \%$ vs PD-LI expression $\geq 10 \%$. (D) PD-LI expression $\geq 50 \%$.

Abbreviations: HR, hazard ratio; OS, overall survival; PD-I, programmed cell death I; PD-LI, programmed cell death ligand-I.

0.69 (95\% CI $0.61-0.77 ; P<0.00001)$ without heterogeneity $\left(P=0.23, I^{2}=29 \%\right)$. PD-1/PD-L1 inhibitor therapy significantly improved OS in the ever-smoked (current or former) subgroup (Figure 4A). In the never-smoked subgroup, four studies were included in the analysis. Three trials enrolled NSCLC patients and one enrolled urothelial carcinoma patients. The pooled HR for OS was 0.88 (95\% CI 0.70-1.11; $P=0.28)$ without heterogeneity $\left(P=0.32, I^{2}=14 \%\right)$. Patients without smoking history could not get significantly OS benefits from PD-1/PD-L1 inhibitor therapy. Compared with nonsmokers, there was a tendency for PD-1/PD-L1 inhibitor to improve OS in smokers ( $P=0.06, I^{2}=71.7 \%$; Figure $\left.4 \mathrm{~A}\right)$. In the $<65$ years subgroups, nine studies reported OS data. Two trials enrolled melanoma patients, four enrolled NSCLC patients, one enrolled urothelial carcinoma patients, one enrolled head and neck carcinoma patients, and one study enrolled renal cell carcinoma patients. The heterogeneity between studies was insignificant $\left(P=0.34, I^{2}=11 \%\right.$ ), and a fixed-effects model was used to pool HR for OS. Patients aged $<65$ years could get OS benefits from PD-1/PD-L1 inhibitor therapy (HR $=0.70,95 \%$ CI $0.63-0.78 ; P<0.00001$; Figure $4 \mathrm{~B}$ ). In the $\geq 65$ years subgroups, five studies were included in the analysis, and no significant heterogeneity was observed $\left(P=0.69, I^{2}=0 \%\right)$. The pooled HR using a random-effect model for OS was 0.74 (95\% CI 0.64-0.85; $P<0.0001)$. There were significant improvements in OS for patients aged $\geq 65$ years (Figure $4 \mathrm{~B}$ ). However, in the $\geq 75$ years subgroups, four studies were included in the analysis, and substantial heterogeneity was observed ( $P=0.01, I^{2}=74 \%$ ). The pooled HR used random-effect model for OS. No significant improvement in OS was observed $(\mathrm{HR}=0.86 ; 95 \% \mathrm{CI} 0.40-1.84 ; P=0.70)$ in patients aged $\geq 75$ years (Figure 4B). For $\mathrm{PS}=0$ population, seven studies reported OS data without heterogeneity $\left(P=0.10, I^{2}=44 \%\right)$. 
A

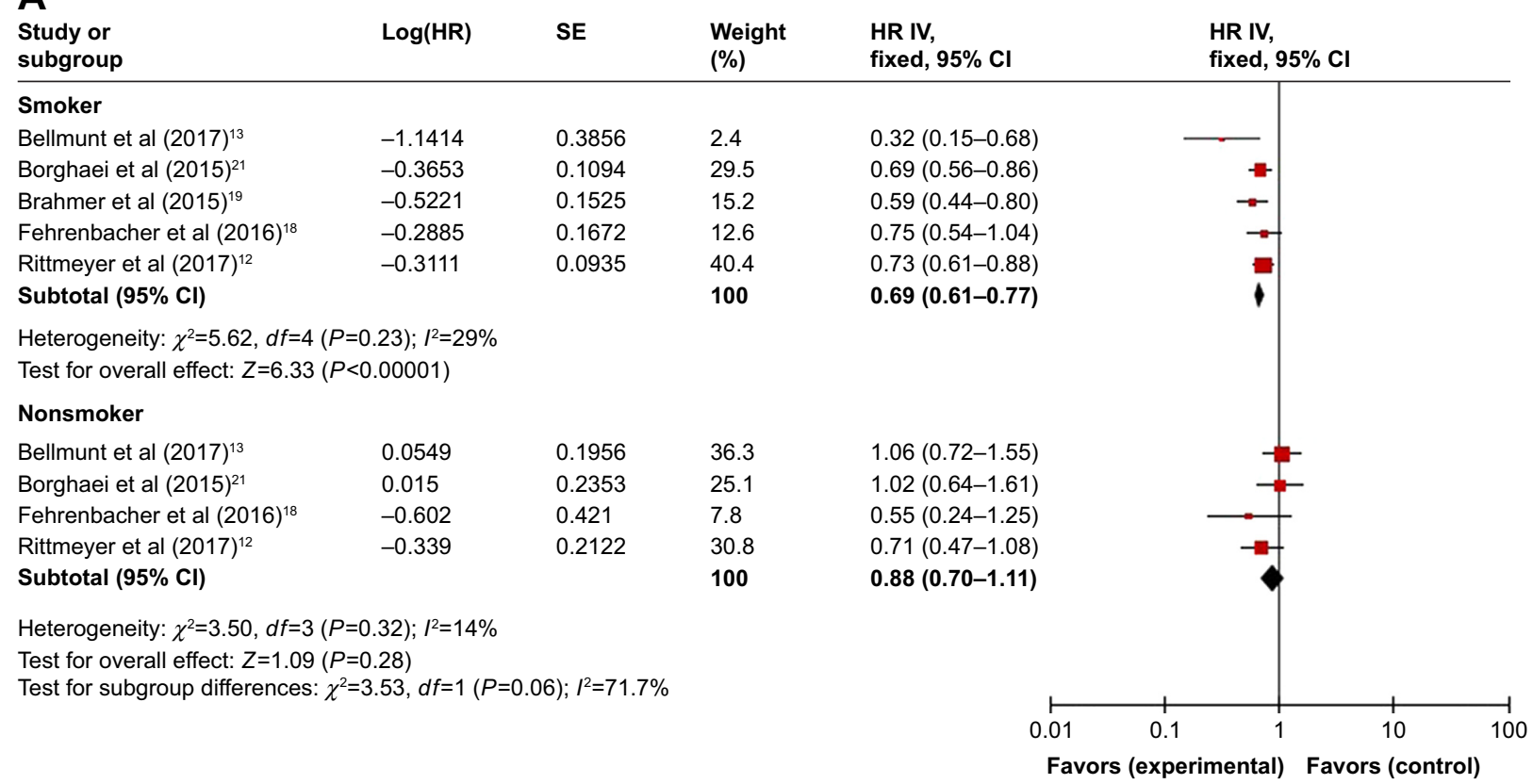

B

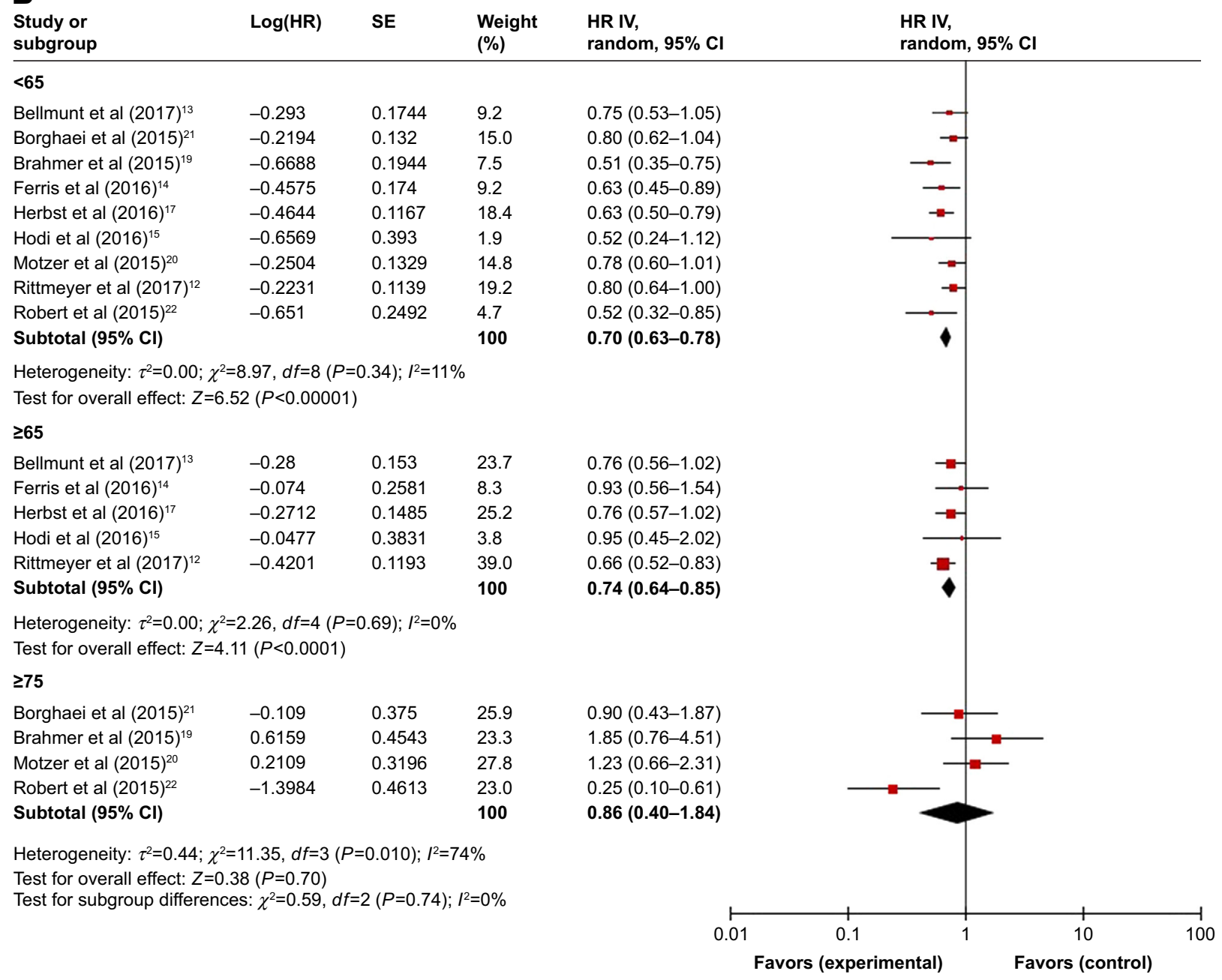

Figure 4 Forest plot for the subgroup analyses of HR of OS for PD-I or PD-LI inhibitors by smoking status and age.

Notes: (A) Smoking status subgroup. (B) Age subgroup.

Abbreviations: HR, hazard ratio; OS, overall survival; PD-I, programmed cell death I; PD-LI, programmed cell death ligand-I. 
For $\mathrm{PS} \geq 1$ population, eight trials reported OS data without heterogeneity $\left(P=0.70, I^{2}=0 \%\right)$. Compared with $P S \geq 1$ population, there was no significant improvement in OS for $\mathrm{PS}=0$ population $\left(P=0.68, I^{2}=0 \%\right)$. Treatment effect was evaluable with data available on sex status from eight trials. There was no significant difference of OS between male and female $\left(P=0.60, I^{2}=0 \%\right)$.

\section{Subgroup analyses by disease factors}

Subgroup analyses were conducted based on disease factors such as histology, line of therapy, and treatment type. In the first-line therapy subgroup, three studies were included in the analysis, and no significant heterogeneity was observed $\left(P=0.16, I^{2}=45 \%\right)$. The pooled HR using a fixed-effects model for OS was 0.53 (95\% CI 0.42-0.67; $P<0.00001)$. Patients could get OS benefits from PD-1/PD-L1 inhibitor as the first-line therapy (Figure 5). In the second line or later treatment, there was also obvious improvement in OS (HR=0.71; 95\% CI 0.66-0.77; $P<0.00001)$ without heterogeneity $\left(P=0.91, I^{2}=0 \%\right)$. The result of test for subgroup differences showed that the first-line therapy had better OS than second line or later treatment $\left(P=0.02, I^{2}=82 \%\right.$; Figure 5). The solid tumors were classified histologically as squamous NSCLC, non-squamous NSCLC, melanoma, urothelial carcinoma, head and neck carcinoma, and renal cell carcinoma, with treatment effect evaluable from all included trials. There was no significant difference of OS between different types of tumors $\left(P=0.89, I^{2}=0 \%\right.$; Figure 6$)$. Nine trials used PD-1 inhibitor, and two studies used PD-L1 inhibitor. The result of test for subgroup differences showed that there was no significant difference between PD-1 inhibitor and PD-L1 inhibitor $\left(P=0.36, I^{2}=0 \%\right)$.

\section{Risk of bias and publication bias}

For almost all studies enrolled in this meta-analysis, low risk of bias existed for all key domains. Only nine trials with open-label design were at high risk of bias (Figure 7).

Funnel plot, Begg's test, and Egger's test were performed to assess the publication bias of literature. The funnel plots did not demonstrate the obvious asymmetry for OS (Figure 8). In addition, Begg's $(P=0.087)$ test and Egger's $(P=0.150)$ test also did not provide any evidence of publication bias for OS. Therefore, no significant publication bias was detected for any of the measured outcomes.

\section{Discussion}

To the best of our knowledge, this review, which included eleven RCTs, is the first meta-analysis that evaluates the impacts of various clinical and molecular characteristics on the efficacy of PD-1/PD-L1 inhibitors for solid tumors. Our meta-analysis found that PD-1/PD-L1 inhibitor therapy significantly improved the OS for patients with melanoma,

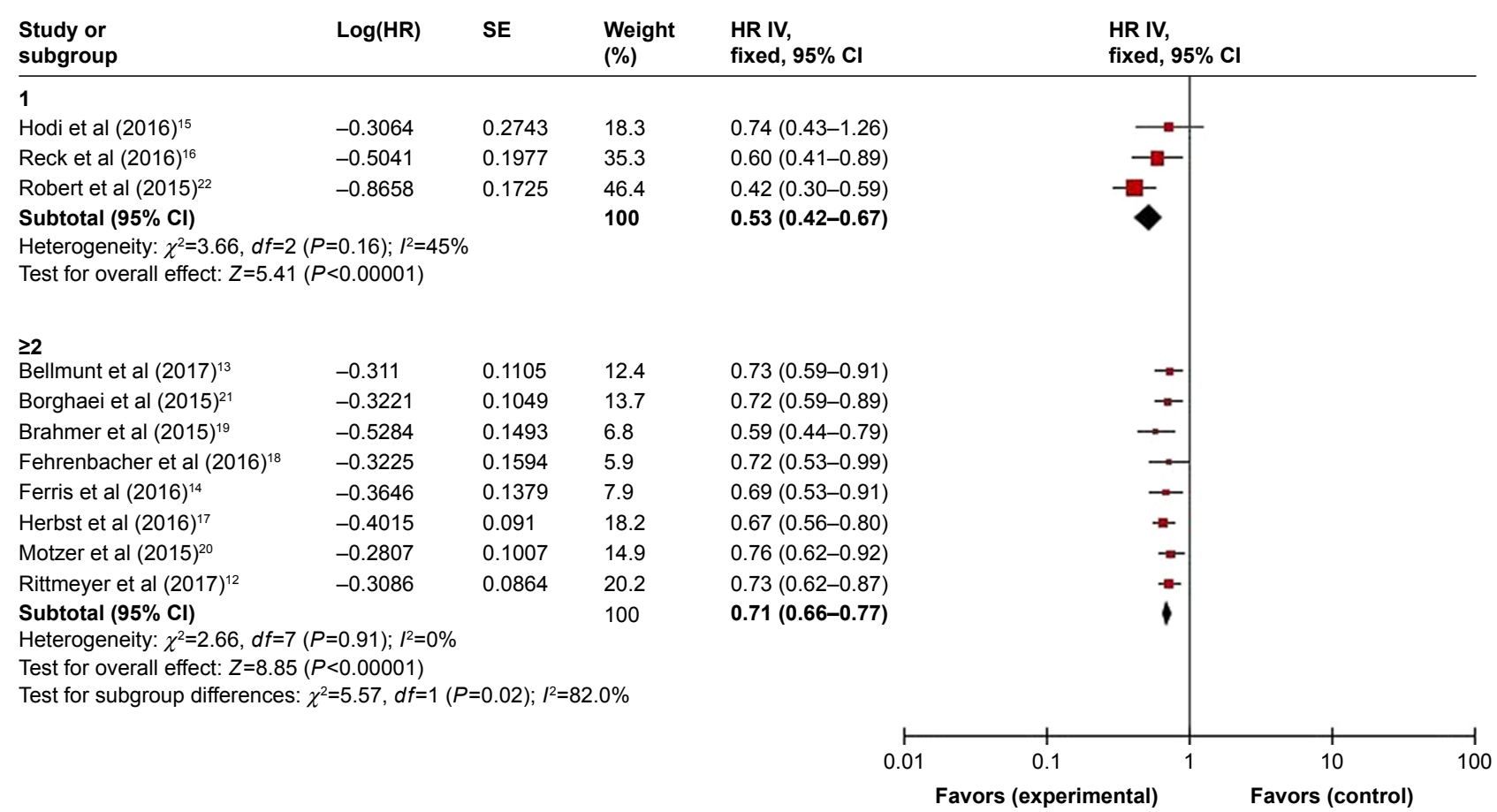

Figure 5 Forest plot for the subgroup analyses of HR of OS for PD-I or PD-LI inhibitors by line of treatment.

Abbreviations: HR, hazard ratio; OS, overall survival; PD-I, programmed cell death I; PD-LI, programmed cell death ligand-I. 


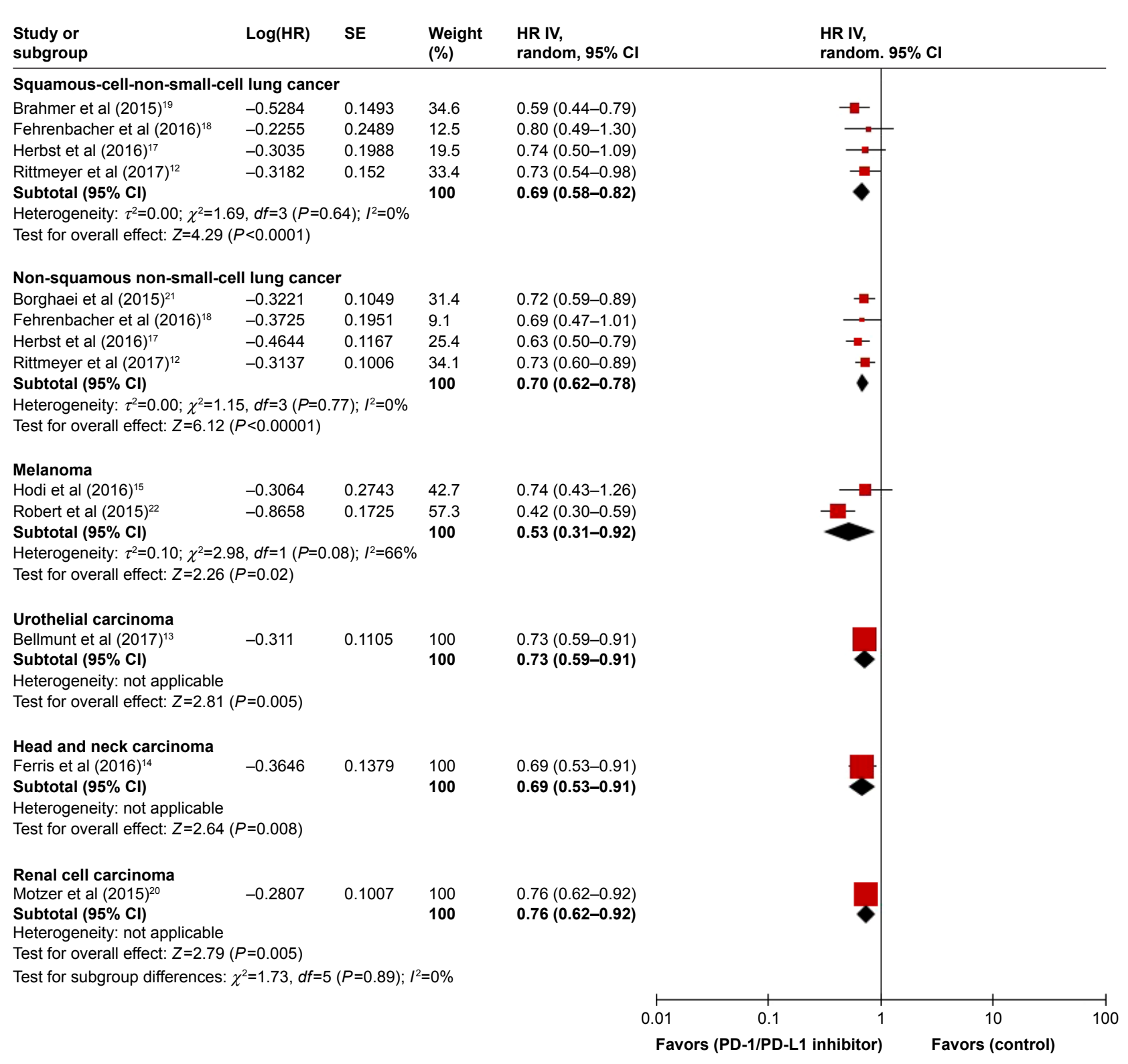

Figure 6 Forest plot for the subgroup analyses of HR of OS for PD-I or PD-LI inhibitors by histology of tumors.

Abbreviations: HR, hazard ratio; OS, overall survival; PD-I, programmed cell death I; PD-LI, programmed cell death ligand-I.

NSCLC, urothelial carcinoma, head and neck carcinoma, and renal cell carcinoma. In addition, PD-L1-positive patients could achieve OS benefits from PD-1/PD-L1 inhibitors regardless of PD-L1 expression level, and a possible doseeffect relationship between the expression of PD-L1 and the OS benefit from PD-1/PD-L1 inhibitors was observed. Patients with higher PD-L1 expression might achieve greater OS benefits. There was an OS improvement for patients with a smoking history, but no OS benefit was observed for nonsmokers. Moreover, compared with second- or laterline treatment, there were better OS benefits in the first-line treatment subgroup. No significant improvement of OS was observed in patients aged $\geq 75$ years. The relative treatment efficacy was similar according to sex (male vs female), PS ( 0 vs $\geq 1$ ), tumor histology (squamous NSCLC vs nonsquamous NSCLC vs melanoma vs urothelial carcinoma vs head and neck carcinoma vs renal cell carcinoma), and treatment type (PD-1 inhibitor vs PD-L1 inhibitor).

$\mathrm{PD}-\mathrm{L} 1$ expression is controversial in predicting the efficacy of response to anti-PD-1/PD-L1 immunotherapy. A previous study showed that the OS benefit of PD-1 inhibitors was limited to the PD-L1 $>1 \%$ population. ${ }^{3}$ However, another meta-analysis indicated that a proportion of PD-L1-negative patients also had an objective response rate improvement from anti-PD-1 therapy. ${ }^{23-25}$ Due to an insufficient number of trials and patients in previous studies, 


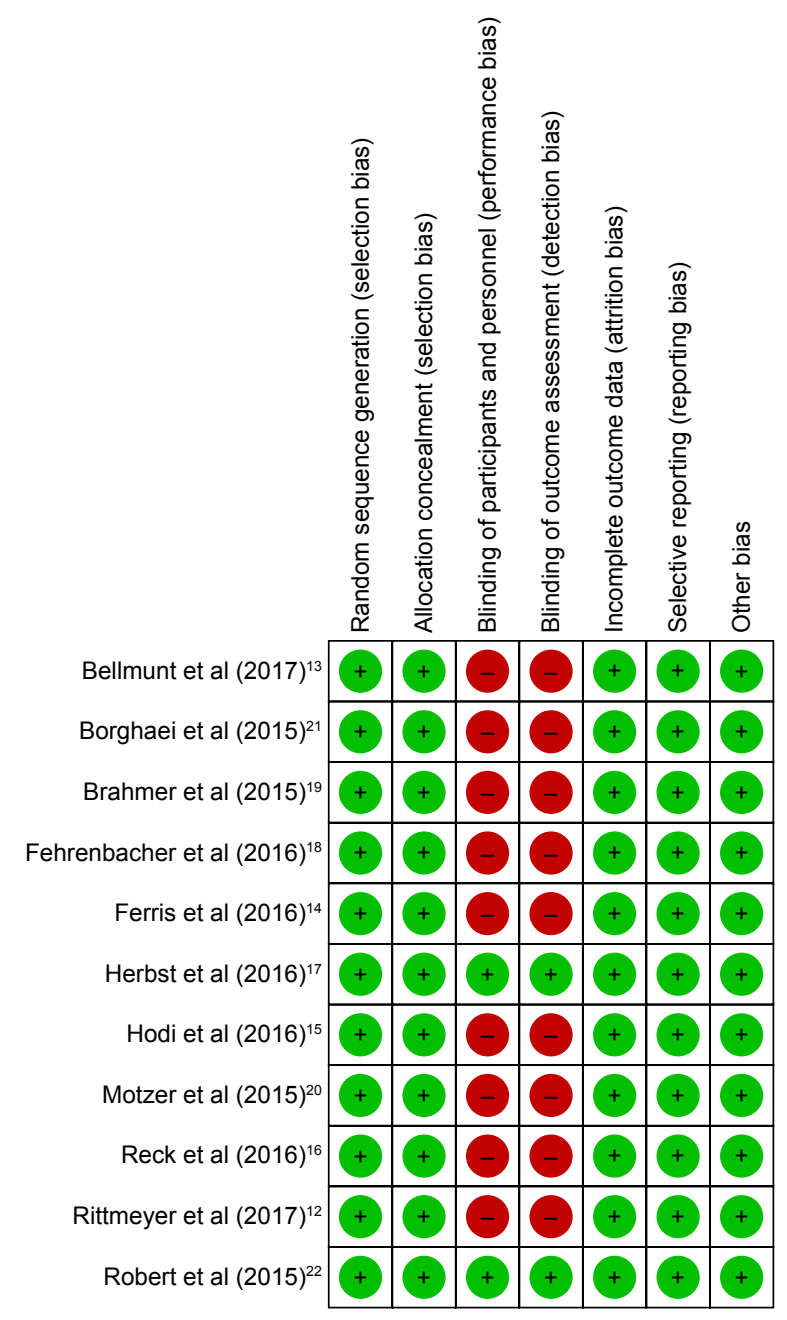

Figure 7 Risk of bias graph.

no consensus had been reached. ${ }^{26,27}$ In our meta-analysis, all the patients with PD-L1-positive tumors could get OS benefits from PD-1/PD-L1 inhibitors regardless of PD-L1 expression level, and a possible dose-effect relationship

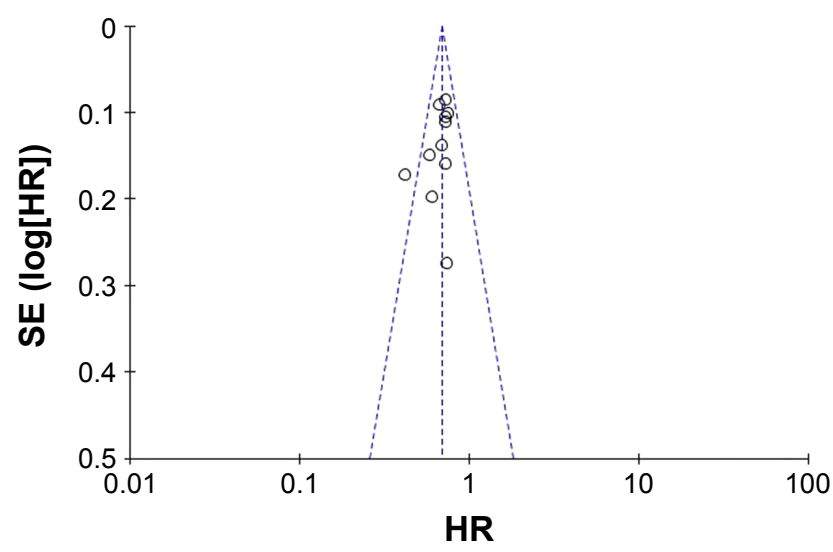

Figure 8 Funnel plot for publication bias. Abbreviations: HR, Hazard ratio; SE, standard error. between the expression of PD-L1 and OS benefit from PD-1/ PD-L1 inhibitors was observed. Based on these results, PD-L1 positive is a valuable biomarker that can predict the efficacy of PD-1/PD-L1 inhibitors and patients with higher PD-L1 expression might achieve greater OS benefits. However, PD-L1 expression assays are affected by several factors, including specimen size, biopsy location, variable components of the tumor and immune microenvironment, and tumor transformation. In addition, the expression of PD-L1 is heterogeneous and dynamic in time and space of different scales. ${ }^{28-30}$ The evaluation at a single time point may not reflect an evolving immune response or accurately predict the response to PD-1/PD-L1 pathway blockades. Moreover, there are no uniform detection assays and standard definition of the cutoff in different trials. ${ }^{31,32}$ These factors will limit the clinical application of PD-L1 expression. Future investigations should focus on standardized and dynamical detection of PD-L1 expression to guide clinical decisions.

Aging is associated with several structural and functional changes in the immune system, which are classified under the term "immunosenescence". ${ }^{33}$ Preclinical and clinical data show that advanced age is associated with decreasing antitumor immune responses, which raises the concern that advanced age may impair the response to immunotherapies. ${ }^{34-37}$ Nonetheless, previous meta-analyses demonstrated that patients aged $>65$ years treated with the ICI have similar clinical efficacy and safety as patients aged $<65$ years, but patients aged $>75$ years may be the tipping point in clinical antitumor immunity. ${ }^{33,38,39}$ Our metaanalysis provided an up-to-date assessment of PD-1/PD-L1 in elderly subjects, and the results were similar to previous studies. No significant improvement in OS was observed in patients aged $\geq 75$ years. Thus, the current data of our analyses did not support the large-scale clinical application of PD-1/PD-L1 inhibitors in patients aged $>75$ years. Exploring the molecular and immune mechanisms, underlying a potential lack of benefit during immunotherapy in elderly patients, represents a tough challenge for future study in this field of cancer treatment.

The tumor genomic landscape is obviously distinct in never smokers compared to smokers, with an average mutation frequency more than tenfold higher in smokers than in never smokers. ${ }^{40}$ Preclinical and clinical data show that higher mutational and neoantigen burdens are associated with durable clinical benefit. ${ }^{41,42}$ Smokers have been hypothesized to get benefits from ICI. However, previous meta-analysis showed that no significant difference of treatment benefit in NSCLC was observed between smokers and never smokers. ${ }^{11}$ 
Herein, with additional clinical trials as compared with the previous study, our meta-analysis found that there was an OS improvement for patients with a smoking history, but no OS benefit was observed for those without a smoking history. Thus, smoking status is a robust clinical biomarker for the selection of PD-1/PD-L1 inhibitors. In addition, related studies have been conducted to assess the effects of smoking on the mutational landscape and identify the molecular signature of smoking. The frequency of $\mathrm{C}>\mathrm{A}$ transversions was defined as a smoking genetic signature that is highly correlated with both elevated mutation burden and clinical benefit with PD-1/PD-L1 inhibitors. Obviously, the mutational smoking signature that provides a more objective and quantitative determination of tobacco carcinogen-induced DNA damage is a far more potential predictor of clinical benefit than a smoking history. With the increasing rapidity and decreased cost of exome-based analyses, this approach could provide a more granular predictor of response to PD-1/PD-L1 inhibitors than immunohistochemistry-based analyses alone.

In addition, our results confirmed that the line of treatment could be used to predict the efficacy of PD-1/PD-L. Despite that first-line and later line of treatment achieved OS improvement, there was a better OS benefit in the first-line treatment subgroup. The hallmark mechanisms of cancer immunotherapy included immunogenic cell death, antigen release and presentation, priming of $\mathrm{T}$ cell responses, enhancement of T cell activity, infiltration into tumor tissues, and depletion of compensatory immunosuppression. Any dysfunction in any of the abovementioned steps will lead to ineffective immunotherapy. Thus, normal immune function and physical condition are significant for the efficacy of immunotherapy. Potential immunosuppressive effects and serious adverse reactions of previously traditional chemotherapy or radiotherapy might negatively affect the efficacy of a later line of immunotherapy. Therefore, our results recommend using immunotherapy as first-line treatment.

There are also some limitations in this meta-analysis. First, there were only eleven trials that met the eligibility criteria, and the number of patients who were included in those trials was low. Second, the control group regimens between those trials are different, which contribute to the increase in the heterogeneity of the meta-analysis. In addition, the nonconformity of PD-L1 assessment between studies caused limited clinical guidance significance. For instance, in our meta-analysis, tumor samples were obtained from pretreatment (archival or recent) tumor biopsy specimens to determine PD-L1 expression in eight studies. The remaining three studies did not report the relevant information. PD-L1 expression was assessed at a central laboratory with the VENTANA SP142 PD-L1 immunohistochemistry assay (Ventana Medical Systems, Inc., Tucson, AZ, USA) in two clinical trials. Six studies evaluated tumor PD-L1 expression by means of immunohistochemical testing (Dako Denmark A/S, Glostrup, Denmark) with the use of a rabbit antihuman PD-L1 antibody (clone 28-8; Epitomics Inc, Burlingame, CA, USA). Two other studies used the immunohistochemistry assay (Dako Denmark A/S) with the murine 22C3 antihuman PD-L1 antibody (Merck \& Co., Inc., Whitehouse Station, NJ, USA). These factors should be carefully considered when employing PD-L1 as a predictive biomarker in clinical practice. Moreover, most of studies included in our analysis consisted primarily of the PD-L1-positive population, which may explain the widely efficacious PD-1/PD-L1 inhibitors in our analysis. This might easily be misleading in clinical application of PD-1/PD-L1 inhibitors. Finally, nine trials used an open-label design that might result in the biased outcome. Further study of updated information is still required.

\section{Conclusion}

Our meta-analysis found that PD-1/PD-L1 inhibitors significantly improved the OS for patients with melanoma, NSCLC, urothelial carcinoma, head and neck carcinoma, and renal cell carcinoma. PD-L1-positive tumors, smoking history, and first-line treatment were potential factors for the efficacy of PD-1/PD-L1 inhibitors. Patients with higher PD-L1 expression might achieve greater OS benefits. In addition, sex, PS, tumor histology, and treatment type could not predict the efficacy of this therapy. In contrast, patients aged $>75$ years and nonsmokers might not get OS benefits from this treatment. These findings can help to predict a better response to PD-1/PD-L1 inhibitors and patient selection in clinical practice.

\section{Data availability statement}

All data are fully available within the paper without restriction.

\section{Acknowledgments}

The authors thank the reviewers for their helpful comments on this article. This review was funded by the National Natural Science Foundation of China (NNSFC; 81670123 and 81770169) and Hubei Province Health and Family Planning Scientific Research Project (WJ2017Q007). The authors thank LetPub (http://www.letpub.com) for its linguistic assistance during the preparation of this manuscript. Yi Ming Weng and Min Peng are the co-first authors. 


\section{Author contributions}

All authors contributed toward data analysis, drafting and revising the paper, gave final approval of the version to be published, and agree to be accountable for all aspects of the work.

\section{Disclosure}

The authors report no conflicts of interest in this work.

\section{References}

1. Sharma P, Allison JP. The future of immune checkpoint therapy. Science. 2015;348(6230):56-61.

2. Emens LA, Ascierto PA, Darcy PK, et al. Cancer immunotherapy: opportunities and challenges in the rapidly evolving clinical landscape. Eur J Cancer. 2017;81:116-129.

3. Maleki Vareki S, Garrigós C, Duran I. Biomarkers of response to PD-1/ PD-L1 inhibition. Crit Rev Oncol Hematol. 2017;116:116-124.

4. Ma W, Gilligan BM, Yuan J, Li T. Current status and perspectives in translational biomarker research for PD-1/PD-L1 immune checkpoint blockade therapy. J Hematol Oncol. 2016;9(1):47.

5. Wang J, Yuan R, Song W, Sun J, Liu D, Li Z. PD-1, PD-L1 (B7-H1) and tumor-site immune modulation therapy: the historical perspective. J Hematol Oncol. 2017;10(1):34.

6. Liu B, Song Y, Liu D. Recent development in clinical applications of PD-1 and PD-L1 antibodies for cancer immunotherapy. J Hematol Oncol. 2017;10(1):174.

7. Postow MA, Sidlow R, Hellmann MD. Immune-related adverse events associated with immune checkpoint blockade. $N$ Engl J Med. 2018;378(2):158-168.

8. Wolchok JD, Chiarion-Sileni V, Gonzalez R, et al. Overall survival with combined nivolumab and ipilimumab in advanced melanoma N Engl J Med. 2017;377(14):1345-1356.

9. Diggs LP, Hsueh EC. Utility of PD-L1 immunohistochemistry assays for predicting PD-1/PD-L1 inhibitor response. Biomark Res. 2017;5:12.

10. Liu D, Wang S, Bindeman W. Clinical applications of PD-L1 bioassays for cancer immunotherapy. J Hematol Oncol. 2017;10(1):110.

11. Lee CK, Man J, Lord S, et al. Clinical and molecular characteristics associated with survival among patients treated with checkpoint inhibitors for advanced non-small cell lung carcinoma: a systematic review and meta-analysis. JAMA Oncol. 2018;4(2):210-216.

12. Rittmeyer A, Barlesi F, Waterkamp D, et al; OAK Study Group. Atezolizumab versus docetaxel in patients with previously treated non-small-cell lung cancer (OAK): a phase 3, open-label, multicentre randomised controlled trial. Lancet. 2017;389(10066):255-265.

13. Bellmunt J, de Wit R, Vaughn DJ, et al; KEYNOTE-045 Investigators. Pembrolizumab as second-line therapy for advanced urothelial carcinoma. N Engl J Med. 2017;376(11):1015-1026.

14. Ferris RL, Blumenschein G, Fayette J, et al. Nivolumab for recurrent squamous-cell carcinoma of the head and neck. $N$ Engl J Med. 2016; 375(19):1856-1867.

15. Hodi FS, Chesney J, Pavlick AC, et al. Combined nivolumab and ipilimumab versus ipilimumab alone in patients with advanced melanoma: 2-year overall survival outcomes in a multicentre, randomised, controlled, phase 2 trial. Lancet Oncol. 2016;17(11):1558-1568.

16. Reck M, Rodríguez-Abreu D, Robinson AG, et al; KEYNOTE-024 Investigators. Pembrolizumab versus chemotherapy for PD-L1-positive non-small-cell lung cancer. N Engl J Med. 2016;375(19):1823-1833.

17. Herbst RS, Baas P, Kim DW, et al. Pembrolizumab versus docetaxel for previously treated, PD-L1-positive, advanced non-small-cell lung cancer (KEYNOTE-010): a randomised controlled trial. Lancet. 2016;387(10027):1540-1550.

18. Fehrenbacher L, Spira A, Ballinger M, et al; POPLAR Study Group. Atezolizumab versus docetaxel for patients with previously treated non-small-cell lung cancer (POPLAR): a multicentre, open-label, phase 2 randomised controlled trial. Lancet. 2016;387(10030):1837-1846.
19. Brahmer J, Reckamp KL, Baas P, et al. Nivolumab versus docetaxel in advanced squamous-cell non-small-cell lung cancer. NEngl JMed.2015; 373(2):123-135.

20. Motzer RJ, Escudier B, Mcdermott DF, et al. Nivolumab versus everolimus in advanced renal-cell carcinoma. $N$ Engl J Med. 2015;373(19): 1803-1813.

21. Borghaei H, Paz-Ares L, Horn L, et al. Nivolumab versus docetaxel in advanced nonsquamous non-small-cell lung cancer. NEngl JMed. 2015; 373(17):1627-1639.

22. Robert C, Long GV, Brady B, et al. Nivolumab in previously untreated melanoma without BRAF mutation. $N$ Engl J Med. 2015;372(4): 320-330.

23. Abdel-Rahman O. Correlation between PD-L1 expression and outcome of NSCLC patients treated with anti-PD-1/PD-L1 agents: a metaanalysis. Crit Rev Oncol Hematol. 2016;101:75-85.

24. Gandini S, Massi D, Mandalà M. PD-L1 expression in cancer patients receiving anti PD-1/PD-L1 antibodies: a systematic review and metaanalysis. Crit Rev Oncol Hematol. 2016;100:88-98.

25. Passiglia F, Bronte G, Bazan V, et al. PD-L1 expression as predictive biomarker in patients with NSCLC: a pooled analysis. Oncotarget. 2016;7(15):19738-19747.

26. Gibney GT, Weiner LM, Atkins MB. Predictive biomarkers for checkpoint inhibitor-based immunotherapy. Lancet Oncol. 2016;17(12): e542-e551.

27. Meng X, Huang Z, Teng F, Xing L, Yu J. Predictive biomarkers in PD-1/PD-L1 checkpoint blockade immunotherapy. Cancer Treat Rev. 2015;41(10):868-876.

28. Taube JM, Anders RA, Young GD, et al. Colocalization of inflammatory response with B7-h1 expression in human melanocytic lesions supports an adaptive resistance mechanism of immune escape. Sci Transl Med. 2012;4(127):127ra37.

29. Madore J, Vilain RE, Menzies AM, et al. PD-L1 expression in melanoma shows marked heterogeneity within and between patients: implications for anti-PD-1/PD-L1 clinical trials. Pigment Cell Melanoma Res. 2015;28(3):245-253.

30. Mukherji D, Jabbour MN, Saroufim M, et al. Programmed death-ligand 1 expression in muscle-invasive bladder cancer cystectomy specimens and lymph node metastasis: a reliable treatment selection biomarker? Clin Genitourin Cancer. 2016;14(2):183-187.

31. Hansen AR, Siu LL. PD-L1 testing in cancer: challenges in companion diagnostic development. JAMA Oncol. 2016;2(1):15-16.

32. Patel SP, Kurzrock R. PD-L1 expression as a predictive biomarker in cancer immunotherapy. Mol Cancer Ther. 2015;14(4):847-856.

33. Ferrara R, Mezquita L, Auclin E, Chaput N, Besse B. Immunosenescence and immunecheckpoint inhibitors in non-small cell lung cancer patients: does age really matter? Cancer Treat Rev. 2017;60: $60-68$.

34. Linton PJ, Dorshkind K. Age-related changes in lymphocyte development and function. Nat Immunol. 2004;5(2):133-139.

35. Goronzy JJ, Weyand CM. Immune aging and autoimmunity. Cell Mol Life Sci. 2012;69(10):1615-1623.

36. Betof AS, Nipp RD, Giobbie-Hurder A, et al. Impact of age on outcomes with immunotherapy for patients with melanoma. Oncologist. 2017;22(8):963-971.

37. Helissey C, Vicier C, Champiat S. The development of immunotherapy in older adults: new treatments, new toxicities? J Geriatr Oncol. 2016; 7(5):325-333.

38. Nishijima TF, Muss HB, Shachar SS, Moschos SJ. Comparison of efficacy of immune checkpoint inhibitors (ICIs) between younger and older patients: a systematic review and meta-analysis. Cancer Treat Rev. 2016;45:30-37.

39. Daste A, Domblides C, Gross-Goupil M, et al. Immune checkpoint inhibitors and elderly people: a review. Eur J Cancer. 2017;82: $155-166$.

40. Govindan R, Ding L, Griffith M, et al. Genomic landscape of non-small cell lung cancer in smokers and never-smokers. Cell. 2012;150(6): $1121-1134$. 
41. Lee W, Jiang Z, Liu J, et al. The mutation spectrum revealed by paired genome sequences from a lung cancer patient. Nature. 2010;465(7297): 473-477.

42. Rizvi NA, Hellmann MD, Snyder A, et al. Cancer immunology. Mutational landscape determines sensitivity to PD-1 blockade in non-small cell lung cancer. Science. 2015;348(6230):124-128.
43. Moher D, Liberati A, Tetziaff J, Altman DG; The PRISMA Group (2009). Preferred Reporting Items for Systematic Reviews and MetaAnalyses. The PRISMA statement. PLoS Med. 6(7):e1000097.

\section{Publish your work in this journal}

OncoTargets and Therapy is an international, peer-reviewed, open access journal focusing on the pathological basis of all cancers, potential targets for therapy and treatment protocols employed to improve the management of cancer patients. The journal also focuses on the impact of management programs and new therapeutic agents and protocols on

patient perspectives such as quality of life, adherence and satisfaction. The manuscript management system is completely online and includes a very quick and fair peer-review system, which is all easy to use. Visit http://www.dovepress.com/testimonials.php to read real quotes from published authors. 